\title{
Exploring Vibrational and Valence Loss Spectra from Oxide Nanoparticles
}

\author{
P. A. Crozier ${ }^{1}$, Qianlang Liu ${ }^{1}$, Kartik Venkatraman ${ }^{1}$, Diane M. Haiber ${ }^{1}$, William J. Bowman ${ }^{1}$, Katia \\ $\mathrm{March}^{2}$ and Peter Rez ${ }^{3}$ \\ 1. School for Engineering of Matter, Transport and Energy, Arizona State University, Tempe, AZ, USA. \\ 2. LeRoy Erying Center for Solid State Science, Arizona State University, Tempe, AZ, USA. \\ ${ }^{3 .}$ Department of Physics, Arizona State University, Tempe, AZ, USA.
}

Monochromated electron energy-loss spectroscopy (EELS) now offers energy resolutions of $\sim 10 \mathrm{meV}$ allowing unprecedented probing of the visible and infrared regions of the spectrum [1]. Features associated with electronic excitations such as plasmons, bandgap measurement, bandgap states and surface states can potentially be probed with spatial resolutions on the order of a few nanometers [2]. Vibrational fingerprints associated with surface layers and molecular adsorbates, as well as phonon modes can now be observed $[1,3,4]$. However, there are many fundamental questions regarding the nature and origin of spectral features observed below the bandgap, which must be understood in order to extract useful information on materials' chemical, optical, and electronic properties. For example, spatial resolution, sensitivity, guided light modes and relativistic effects must be carefully considered in the interpretation of the spectra. Comparison with optical spectroscopies such as infrared/visible/UV absorption and Raman spectroscopy is also helpful.

Oxide nanoparticles are an important class of materials with applications to catalysis, sensors and fuel cells. Electronic states associated with dopants, defects or surface adsorbates may give rise to spectral intensity within the bulk bandgap. States associated with dopants and surface species have been detected in $\mathrm{Pr}$ doped $\mathrm{CeO}_{2}$ and on hydroxylated $\mathrm{MgO}$ surfaces [2,5]. The aloof beam geometry, where the beam is positioned a few nanometers outside the particle surface, offers the possibility of probing the surface of nanoparticles while minimizing electron beam damage. Figure 1a shows a simulated aloof beam spectrum from a sample consisting of $0.1 \mathrm{~nm}$ of $\mathrm{TiO}_{2}$ on an $\mathrm{MgO}$ substrate. This is a particularly favorable materials combination for EELS but for other systems, the surface/bulk signal ratio is often very low. The surface/bulk signal ratio may be increased by going to higher scattering angles although the total signal strength will drop. Under some circumstances, Cherenkov and guided light modes can give rise to additional intensity or peaks within the bandgap region, which masks the signal associated with electronic excitations. These peaks become more pronounced with increasing particle size, refractive index and incident beam energy. For example, at $60 \mathrm{kV}$, guided light modes are very weak in spectra from isolated $100 \mathrm{~nm} \mathrm{MgO}$ cubes (refractive index 1.7) but dominate the bandgap region in spectra from aggregates of $100 \mathrm{~nm}$ anatase particles (refractive index 2.6) [6]. The interpretation of the valence loss spectrum in terms of electronic structure becomes more straightforward with decreasing particle size and incident beam energy.

Oxide nanoparticles can also be probed with vibrational EELS. To facilitate the spectral interpretation, Raman $(532 \mathrm{~nm})$ and FTIR spectra were recorded from the same materials. Figure 1b displays an aloof beam EELS from a $\mathrm{CeO}_{2}$ cube showing a peak at $61 \mathrm{meV}$ with a FWHM of $20 \mathrm{meV}$. The spectrum looks very similar to the Raman (convolved with a Gaussian broadening function to match the EELS resolution), which shows a peak at $56.5 \mathrm{meV}$ corresponding to the $\mathrm{F}_{2 \mathrm{~g}}$ mode of the fluorite structure. Doping the $\mathrm{CeO}_{2}$ with $\operatorname{Pr}$ (Figure 1c) causes the $\mathrm{F}_{2 \mathrm{~g}}$ peak in the Raman spectrum to shift up to $57.5 \mathrm{meV}$ along with the appearance of a second satellite peak at around $71 \mathrm{meV}$. EELS shows a broad peak centered at $64 \mathrm{meV}$ 
which is lower in energy than the broadened Raman peak at $69 \mathrm{meV}$. Figure $1 \mathrm{~d}$ is from anatase with the Raman showing three peaks with a maximum at around $79 \mathrm{meV}$. The convolved Raman approximately matches the EELS although the relative intensity and position of the satellite peak (at $45-50 \mathrm{meV}$ ) is different in the two spectra. The origin of the peak shifts, for example between the Raman and EELS for pure $\mathrm{CeO}_{2}$, is not well understood at the moment. Comparison with FTIR spectra will also be discussed [7].

References:

[1] O.L. Krivanek et al, Nature 514 (2014) p. 209.

[2] Q. Liu et al, Ultramicroscopy (in press).

[3] P.A. Crozier et al, Ultramicroscopy 169 (2016) p. 30.

[4] D. Haiber et al, (these proceedings)

[5] W.J. Bowman et al, Ultramicroscopy 167 (2016) p. 5.

[6] Q. Liu et al, (these proceedings)

[7] The authors acknowledge support from NSF Graduate Research Fellowship Program (DGE-1311230), NSF DMR 1308085, NSF CHE-1508667 and DOE DE-SC0004954. The authors acknowledge John M. Cowley Center for High Resolution Microscopy at Arizona State University.
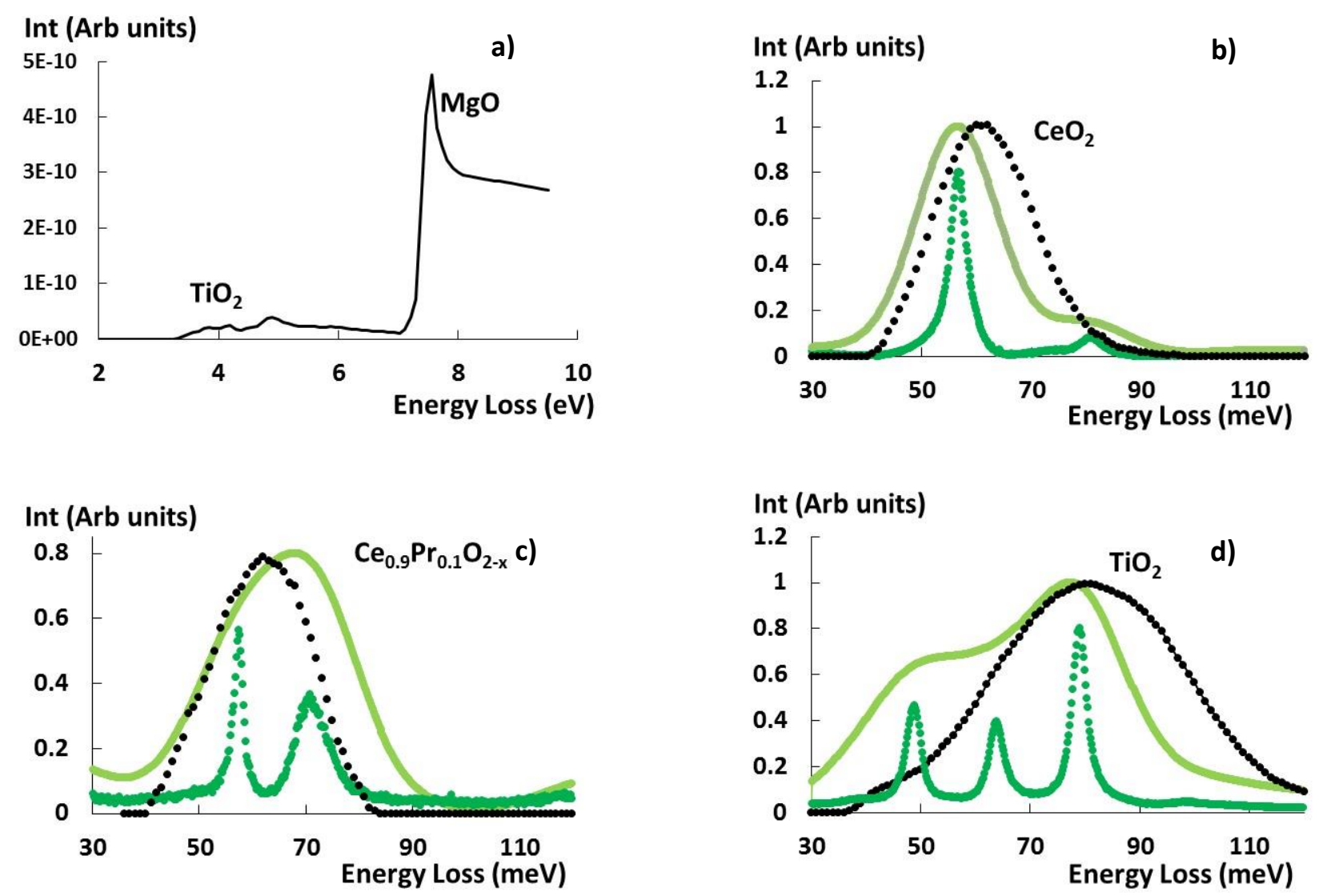

Figure 1. a) Simulation of aloof beam EELS spectrum for $0.1 \mathrm{~nm}$ layer of $\mathrm{TiO}_{2}$ on $\mathrm{MgO}$ substrate. b), c) and d) background subtracted vibrational EELS (black), Raman (dark green) and broadened Raman spectra (light green) from $\mathrm{CeO}_{2}, \mathrm{Ce}_{0.9} \mathrm{Pr}_{0.1} \mathrm{O}_{2-\mathrm{x}}$ and $\mathrm{TiO}_{2}$ (anatase). (EELS energy resolution $\sim 17$ $\mathrm{meV})$. 